Kompass

Autoimmun

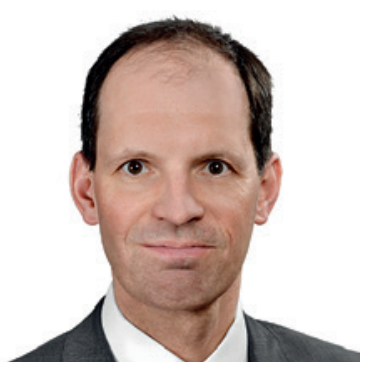

\title{
Der Blick auf die Komorbiditäten zeigt auch die Versorgungsqualität
}

Martin Aringer

Universitätsklinikum Carl Gustav Carus der Technischen Universität Dresden, Medizinische Klinik und Poliklinik III, Dresden, Deutschland

Abstract aus Redeker I, Callhoff J, Hoffmann F, et al.: The prevalence and impact of comorbidities on patients with axial spondyloarthritis: results from a nationwide population-based study. Arthritis Res Ther 2020;22:210.

\section{Keywords}

Axial spondyloarthritis · Comorbidity · Multimorbidity · Disease activity · Functional status

\begin{abstract}
Background: In contrast to other chronic rheumatic musculoskeletal diseases such as rheumatoid arthritis, comorbidities in axial spondyloarthritis (axSpA) and their impact on disease outcomes are less well studied. The aim of this study was to investigate the prevalence of comorbidities and their association with disease activity and functional impairment in a large populationbased cohort of patients with axSpA.
\end{abstract}

Methods: A random sample of patients with axSpA, stratified by age and sex, was drawn from health insurance data. Patients in the sample received a survey on demographic, socioeconomic, and disease-related parameters. Comorbidities were defined using the Elixhauser coding algorithms excluding rheumatoid arthritis/collagen vascular diseases and including osteoporosis and fibromyalgia, resulting in a set of 32 comorbidities. The prevalence of comorbidities in the axSpA patients and their pharmacological treatment were examined. Multivariable linear regression models were calculated to determine the association of comorbidities with disease activity and functional status.

Results: A total of 1776 axSpA patients were included in the analyses (response, 47\%; mean age, 56 years; $46 \%$ female). The most prevalent comorbidities were hypertension, depression, and chronic pulmonary disorders. The number of comorbidities was significantly associated with both the BASDAI and BASFI: $\beta$ (95\% $C I)=0.17(0.09-0.24)$ and $0.24(0.15-0.32)$, respectively. When analysed separately, hypertension, depression, and chronic pulmonary disease were comorbidities with a significant and independent association with BASFI, while for BASDAI, such an association was found for depression and chronic pulmonary disease only.

Conclusions: Comorbidities are common in axSpA patients and are associated with higher disease activity and higher levels of functional impairment. Higher disease activity and higher levels of functional impairment might be indicators of severe disease resulting in the development of comorbidities.

(c) The Author(s) 


\section{Transfer in die Praxis}

Komorbiditäten spielen bei einer Reihe entzündlich-rheumatischer Erkrankungen eine wesentliche Rolle. Bekannte Beispiele dafür sind das deutlich erhöhte kardiovaskuläre Risiko bei systemischem Lupus erythematodes (SLE) und rheumatoider Arthritis (RA), aber auch bei der Gicht, die Depressivität und die Osteoporose.

Bei den axialen Spondylarthritiden (axSpA), also dem Morbus Bechterew und der nichtradiografischen axialen Spondylarthritis, standen Komorbiditäten bisher nicht im Vordergrund. Auch die Datenlage zur Prävalenz solcher Probleme war im Vergleich zu SLE, RA und Gicht viel schlechter. Die jetzt vorliegende Analyse der Berliner Kolleginnen und Kollegen (Redeker et al., Arthritis Res Ther 2020;22:210) stellt komplette und erfreulicherweise für Deutschland direkt anwendbare Daten zur Verfügung, die auch Aussagen zur Versorgungsqualität provozieren.

Für die zugrunde liegende Studie wurden 5000 von fast 22000 Patienten mit axialer Spondylarthritis zufällig ausgewählt und die noch versicherten 4471 Patienten angeschrieben. 2118 Patienten antworteten (47\%) und 2082 davon gaben auch ihre Erlaubnis, die Daten mit den Versicherungsdaten zu verknüpfen. Für die Analyse übrig blieben 1776 Patienten mit dann auch bestätigter axSpADiagnose.

Neben den Fragbogendaten, die unter anderem Aktivität (mittels Bath Ankylosing Spondylitis Disease Activity Index (BASDAI)), Funktion (mittels Bath Ankylosing Spondylitis Functional Index (BASFI)) und Lebensqualität (mittels 5-Item WHO Well-Being Index (WHO5)) beinhalteten, konnten so die Komorbiditäten breit und offen untersucht werden. Dafür wurden die Elixhauser Kodierungsalgorithmen in einer etwas angepassten Form (ergänzt um Osteoporose und Fibromyalgie, aber ohne RA und Kollagenosen) verwendet. Gut die Hälfte der Patienten hatten keine (17\%) oder nur 1 bis 2 Komorbiditäten (41\%), während 25\% 3 bis 4 und 17\% sogar 5 oder mehr Komorbiditäten aufwiesen. Wie erwartet stieg die Zahl der Komorbiditäten mit dem Lebensalter und damit der Erkrankungsdauer und dem Intervall seit der Diagnose an. Im Mittel waren die Patienten ohne Komorbiditäten 45 Jahre, die mit mehr als 4 Komorbiditäten 66 Jahre alt. Entsprechend nahmen auch Vollbeschäftigung (von 54\% auf 6\%) und (beruflicher) Stress (von 46\% auf 25\%) ab.

Fast zwei Drittel verwendeten nichtsteroidale Antirheumatika. Mit der Zahl der Komorbiditäten und dem Lebensalter stiegen Glukokortikoide, blande Analgetika und Physiotherapie an, während die Biologika von 26\% auf 9\% abnahmen. Gleichzeitig stiegen der BASDAI und der BASFI an. Etwas bedenklich stimmt dabei die Tatsache, dass nur bei den jüngeren Patienten ohne Komorbiditäten, die zu immerhin 52\% in rheumatologischer Betreuung waren, der BASDAI-Mittel- wert mit 3,7 noch knapp unter 4 lag und bei allen anderen Gruppen über diesem Wert, der die Schwelle zur Biologika-Indikation darstellt. In der Gruppe mit den meisten Komorbiditäten erreichte er 5,2. Dass diese Gruppe auch zu gut 25\% mit Glukokortikoiden behandelt wurde, erscheint ebenso problematisch.

Wirklich spannend ist aus meiner Sicht aber die Zusammensetzung der gefundenen Komorbiditäten. Am häufigsten war mit 52\% die (in aller Regel unkomplizierte) Hypertonie. 16\% hatten einen Diabetes mellitus, nur 14\% waren übergewichtig. 14\% hatten kardiale Arrhythmien, 6\% eine Herzinsuffizienz und 9\% periphere Gefäßerkrankungen. Etwa 10\% hatten ein Malignom (8,3\% ohne Metastasen, 1,4\% mit Metastasen, 0,6\% Lymphome). Eine chronische Lungenerkrankung bestand bei 23\%, eine Lebererkrankung bei $12 \%$ und eine Niereninsuffizienz bei 8\%. Immerhin etwa ein Viertel (26\%) der Patienten hatten eine Depression, 4\% ein Fibromyalgiesyndrom. Raucher waren in der jüngsten Gruppe ohne Komorbiditäten häufiger vertreten (22\%) und in der Gruppe mit den meisten Komorbiditäten nur mehr zu 14\%. Alkoholmissbrauch (1,6\%) und Drogenmissbrauch $(1,1 \%)$ waren selten.

Und dann sind auch noch die axSpA-assoziierten Erkrankungen auffällig: Uveitis bei 27\%, Psoriasis bei 15\%, chronisch-entzündliche Darmerkrankungen (CED) bei 9\%. Vermutlich sind hier auch noch die Osteoporose (13\%), Herzklappenerkrankungen (immerhin 8\%) und zum Teil auch die Hypothyreose (14\%) einzurechnen.

\section{Fazit}

Auch bei Patienten mit axialen Spondylarthritiden sind Komorbiditäten ein Thema. Kardiovaskuläre Erkrankungen stechen dabei aber nicht wie bei anderen entzündlich-rheumatischen Erkrankungen besonders heraus, wenngleich bei einem mittleren Alter von 56 Jahren etwa die Hälfte an einer Hypertonie litt. Der Anteil der Patienten mit Depression ist mit einem Viertel hingegen beträchtlich und auch eine Malignomrate von 10\% erscheint relativ hoch. Wie erwartet ist die Rate an Osteoporose auf mehr als 10\% erhöht - und Begleiterkrankungen der Spondylarthritis-Gruppe, insbesondere Uveitis, Psoriasis und CED, spielen eine relevante Rolle. Zu hohe BASDAI-Werte und zu viele Patienten unter Glukokortikoiden sprechen für Verbesserungspotenzial in der Versorgung.

\section{Disclosure Statement}

Advisory Boards und/oder Vorträge für AbbVie, MSD, Novartis, Pfizer.

Korrespondenz an:

Martin Aringer, martin.aringer@uniklinikum-dresden.de 